\title{
Efficacy and Toxicity of Induction Therapy with Cladribine, Idarubicin, and Cytarabine (IAC) for Acute Myeloid Leukemia
}

\author{
SUSAN K. WOELICH ${ }^{1}$, JAMES T. BRAUN ${ }^{1}$, MARTIN W. SCHOEN ${ }^{2}$, RESHMA RAMLAL ${ }^{2}$, \\ CARL E. FRETER ${ }^{2}$, PAUL J. PETRUSKA ${ }^{2}$ and JACK M. LIONBERGER ${ }^{2}$ \\ ${ }^{1}$ SSM Saint Louis University Hospital, Saint Louis, MO, U.S.A.; \\ ${ }^{2}$ Saint Louis University School of Medicine, Saint Louis, MO, U.S.A.
}

\begin{abstract}
We report our single-center experience with cytarabine and idarubicin for induction therapy for acute myeloid leukemia (AML) with an additional 5 days of cladribine (IAC therapy). From July 2012 to September 2014, 38 patients completed a full course of IAC induction. Median patient age was 61 years, $61 \%$ of patients were $\geq 60$ years old, and $71 \%$ were male. The complete remission (CR) rate was $63 \%$ following a single induction course, three patients (8\%) required a second induction course to achieve $C R$, for an overall response rate of $71 \%$. The median duration of severe neutropenia was 30.5 days. Thirty-two percent of patients developed mucositis, $76 \%$ experienced diarrhea, and $61 \%$ developed a rash. Incidence of $C R$ following IAC induction therapy for AML was comparable to historical data, but with frequent diarrhea, rash, and fungal infections. This study found IAC efficacy and toxicity was similar irrespective of age.
\end{abstract}

Among adults ( $>18$ years old) who develop acute myeloid leukemia (AML), induction therapy will result in complete remission (CR) in 60-70\%, and about half of these will survive for 3 years or more. However, these numbers do not reflect the complexity added by age, co-morbidity and disease risk classification (1). Importantly, survival is less common and shorter in patients over 60 years of age, for reasons which have not been completely delineated, but likely due in part to the higher proportion of such cases with poor genetic risk features (2). It is significant to note that the median age for AML diagnosis is 66 years, and therefore that the majority of people stricken with AML are older (3).

Correspondence to: Jack M Lionberger, MD, Ph.D., Saint Louis University, 3rd Floor West Pavilion, 3655 Vista Ave, Saint Louis, MO 63110, U.S.A. Tel.: +1 3145778854, Fax: +1 3142681167, e-mail: jlionber@slu.edu

Key Words: Acute myeloid leukemia, AML, induction chemotherapy, cladribine, toxicity, adverse events.
Therapy for AML has been slow to evolve. For over 40 years, standard therapy has included 7 days of cytarabine and 3 days of anthracycline (' $7+3$ ' regimen) (4). Other combinations have been studied in an effort to improve outcomes in older persons, without overwhelming success. However, the Polish Adult Leukemia Group (PALG) reported that adding cladribine to daunorubicin-containing $7+3$ regimen (so called ' $7+3+5$ ' or DAC) increased the complete remission (CR) rate, reduced hospital stay, and did not significantly increase toxicity in a study population of patients younger than 60 years $(5,6)$. The authors concluded that cladribine increases the potency of the $7+3$ regimen, and may improve long-term survival in patients between 40 and 60 year old. National Comprehensive Cancer Network (NCCN) guidelines have recently included DAC as an option for AML induction therapy, albeit in patients under 60 years old (7).

At Saint Louis University Hospital (SLU), DAC has been modified to use idarubicin (IAC) as the preferred anthracycline $(8,9)$. Two retrospective reviews found substitution of daunorubicin by idarubicin in combination with cladribine and cytarabine was safe and effective (10, 11). In contrast with the PALG studies $(5,6)$, SLU routinely utilizes IAC induction in patients over 60 years of age. Here we report the SLU experience with IAC regarding response and toxicity and analyze outcomes as a function of age.

\section{Patients and Methods}

Study design. This was a retrospective study examining AML and high-risk myelodysplasia (i.e. blast count $>10 \%$ ) in patients diagnosed and treated between 7/1/12 and 9/30/14 at SLU Hospital. This study received approval from the SLU Institutional Review Board and SLU Hospital Research Compliance Committee (IRB \# 25011).

Study population. Eligible patients were 18 years or older and had received at least one full cycle of IAC $\left(12 \mathrm{mg} / \mathrm{m}^{2}\right.$ idarubicin $\times 3$ days, $200 \mathrm{mg} / \mathrm{m}^{2}$ cytarabine $\times 7$ days, and $5 \mathrm{mg} / \mathrm{m}^{2}$ cladribine $\times 5$ days). Patients had a diagnosis of AML or high-risk myelodysplasia, and excluded those who: had acute promyelocytic leukemia; were pregnant; had impaired renal (creatinine clearance $<50 \mathrm{ml} / \mathrm{min}$ ), 
hepatic (total bilirubin $>2.5 \mathrm{mg} / \mathrm{dl}$ ), or cardiac (left ventricular ejection fraction $<50 \%$ ) function.

Study outcomes. Primary outcome assessed was response (CR rate) after one or two courses of IAC. Secondary outcome was toxicity, including duration of neutropenia, incidence of mucositis, diarrhea, rash, infections and intensive care unit admissions.

Data collection. The SLU hospital electronic health record (EHR) (EPIC Systems, Verona WI) was searched for patients treated between 7/1/2012 and 9/30/2014 using the International Classification of Diseases ninth revision codes 205.00 (AML) and 208.9 (unspecified leukemia). In cases that preceded the EHR resource, paper charts were manually extracted to identify appropriate patients. Collected data included: baseline serum chemistry and complete blood count values; molecular prognostic indicators (genetic mutations); cytogenetics (karyotype and fluorescent in situ hybridization) data; and blast percentage in peripheral blood and bone marrow. Fourteen-day post-induction and CR bone marrow cytomorphology, flow cytometry; and genetic marker perseverance were recorded.

Regarding adverse events, clinical notes were searched for the terms "diarrhea", "rash", and "mucositis". Additionally, the medication administration record was interrogated to find therapies specific for mucositis or diarrhea, while microbiological and pathological data were assessed for positive culture and skin biopsy data.

CR was defined per World Health Organization classification with $<5 \%$ blasts in bone marrow, absolute neutrophil count (ANC) $>1000$ cells $/ \mathrm{mm}^{3}$, platelet count $>50,000$ cells $/ \mathrm{mm}^{3}$, and hemoglobin $>8 \mathrm{~g} / \mathrm{dl}$, with transfusion independence for $>1$ week (12). Duration of severe neutropenia was defined as the time from the start of therapy to ANC $>500$ cells $/ \mathrm{mm}^{3}$ (13). Duration of neutropenia was defined as the time from the start of therapy until ANC $>1000$ cells $/ \mathrm{mm}^{3}$ (13).

Statistical methods. Outcomes are reported via descriptive statistics. Subgroup analysis of remission rate and toxicities stratified by age was conducted using the chi-squared test. A $p$-value of less than 0.05 was considered significant. IBM SPSS Statistics Version 22.0.0 (IBM Corp., Armonk, NY, USA) was used for statistical analysis.

\section{Results}

Patient and disease characteristics. Thirty-eight patients met the inclusion criteria, out of a total of 48 cases identified. Ten patients were excluded due to abbreviated treatment, non-diagnosis $(<10 \%$ blasts $)$, and therapy outside of the study window. Two out of 38 patients had refractory anemia with excess blasts and one had chronic myelomonocytic leukemia. The patient group was skewed towards male sex $(71 \%)$ and older age (median $=61$ years; range $=20-78$ years; mean $=57.5 \pm 13.7$ years) than that treated by the PALG. Median white blood cell count, marrow blast percentage, cytogenetics and NCCN leukemia risk classification are summarized in Table I. Three patients were classified as having high-risk myelodysplasia (blast count between 10$19 \%$ ), and one patient did not have sufficient data for NCCN leukemia risk stratification (7).
Response rate. Outcomes are summarized in Table II. Twentyseven out of 38 patients (71\%) achieved a CR, 63\% requiring only a single induction course of induction therapy. Another three patients had incomplete CR for an overall response of CR + CRi of $79 \%$. When stratified by leukemia risk, 7/8 (88\%) patients with favorable risk had an overall response, compared to $13 / 14(93 \%)$ with intermediate risk. Only 10/16 (63\%) with unfavorable risk had a response $(p=0.10)$. Fifteen of 38 patients $(39.5 \%)$ were $<60$ years old, of which $87 \%(13 / 15)$ had an overall response. In comparison, overall response was achieved $17 / 23(74 \%)$ of patients $\geq 60$ years of age, $p=0.35$.

Toxicity. Myelosuppression: All patients experienced neutropenia and thrombocytopenia. As shown in Table II, two different neutropenia states were followed in the 33 patients surviving to hospital discharge. ANC $>500 \mathrm{cell} / \mathrm{mm}^{2}$ is regarded as severely neutropenic with high risk of gramnegative rod infection from gut mucosa. A second ANC threshold of 1,000 cells $/ \mathrm{mm}^{2}$ reflects the productivity of a fully recovering marrow.

Rash: During induction therapy, 23/38 patients (61\%) developed skin rash; the average time to first rash (mean $\pm \mathrm{SD}$ ) was $10.2 \pm 4.2$ days. Eight cases were biopsied: one was attributed to trauma, two to hypersensitivity, one to Sweet's syndrome, two to perivascular dermatitis, one to chemotherapy-induced rash, and one to psoriaform dermatitis (Table III).

Gastrointestinal symptoms: Twelve patients (32\%) experienced mucositis, with symptom duration ranging from 2-48 days. Out of the 29 patients experiencing diarrhea, 13 $(45 \%)$ of these suffered longer than 7 days. The average number of days of loperamide administration was $3.6 \pm 0.8$ days (median $=2$ days). Sixteen percent of patients became infected with Clostridium difficile, with $20.8 \%$ occurrence in those aged 60 years or more versus $7.1 \%$ in the younger population $(p=0.264)$ (Table III).

Mucositis: Mucositis was documented in 32\% of patients, with median duration of 11.5 days. Patients received diphenhydramine, aluminum, magnesium, simethicone, viscous lidocaine-containing mouth rinse for a mean of $4.7 \pm 1.9$ days.

Infections: Thirty-seven out of 38 patients $(97 \%)$ had at least one bout of fever during their hospitalization. Infections were bacteria implicated in 53\%, while five patients $(13 \%)$ had fungal infections. Two patients had both bacteria and fungus isolated (Table III). Most common infection was found on blood culture, followed by pneumonia and Clostridium difficile gastrointestinal infection (Table III). Five patients developed vancomycin-resistant enterococcus, in each case cultured from the bloodstream. Stenotrophomonas was twice isolated from the blood and once from the lungs from a bronchial alveolar lavage. Escherichia coli accounted for two cases of bacteremia and one of pneumonia. The three fungal 
Table I. Patient characteristics, $n=38$.

\begin{tabular}{|c|c|c|c|c|}
\hline Patient characteristics & $\mathrm{n}$ & $\%$ & Median & Range \\
\hline Age, years & - & - & 61 & $20-78$ \\
\hline Male gender & 27 & 71 & - & - \\
\hline \multicolumn{5}{|l|}{ Molecular markers } \\
\hline FLT3 ITD & 5 & 13 & - & - \\
\hline FLT3 TKD & 1 & 3 & - & - \\
\hline$N P M 1$ & 8 & 21 & - & - \\
\hline$C E B P A$ & 1 & 3 & - & - \\
\hline \multicolumn{5}{|l|}{ Cytogenetics } \\
\hline Favorable & 4 & 11 & - & - \\
\hline Intermediate & 21 & 55 & - & - \\
\hline Unfavorable & 12 & 32 & - & - \\
\hline Not assessed & 1 & 3 & - & - \\
\hline \multicolumn{5}{|l|}{ NCCN leukemia risk } \\
\hline Favorable & 8 & 21 & - & - \\
\hline Intermediate & 14 & 37 & - & - \\
\hline Poor & 16 & 42 & - & - \\
\hline \multicolumn{5}{|l|}{ Hematological parameters } \\
\hline $\mathrm{WBC}, \times 10^{3} / \mathrm{mm}^{3}$ & - & - & 15.3 & $1.0-220.2$ \\
\hline Hemoglobin, g/dl & - & - & 8.4 & $5.0-13.5$ \\
\hline Platelets, $\times 10^{3} / \mathrm{mm}^{3}$ & - & - & 52.5 & 6-681 \\
\hline ANC, cells $\times 10^{3} / \mathrm{mm}^{3}$ & - & - & 1 & $5-20.5$ \\
\hline Peripheral blood blasts & - & - & 28 & $0-95$ \\
\hline Bone marrow blasts, $\%$ & - & - & 52 & $12-99$ \\
\hline Bone marrow cellularity, $\%$ & - & - & 90 & $60-100$ \\
\hline
\end{tabular}

ANC: Absolute neutrophil count; CEBPA: CCAAT/Enhancer binding protein alpha; FLT3: fms like tyrosine kinase 3; ITD: internal tandem duplication; TKD: tyrosine kinase domain; NPM1: nucleophosmin 1; NCCN: National Comprehensive Cancer Network.

Table II. Hematological recovery and remission outcomes.

\begin{tabular}{lcccc}
\hline Outcome measure & $\mathrm{n}$ & $\%$ & Median & Range \\
\hline CR & & & & \\
$\quad$ CR first induction & 24 & 63 & - & - \\
CR second induction & 3 & 8 & - & - \\
Overall CR & 27 & 71 & - & - \\
CRi & 3 & 8 & - & - \\
Response rate $(\mathrm{CR}+\mathrm{CRi})$ & 30 & 79 & - & - \\
CR by age & & & & - \\
$<60$ Years & $12 / 15$ & $80 *$ & - & - \\
$\geq 60$ Years & $15 / 23$ & 65 & - & - \\
CR by NCCN leukemia risk & & & & - \\
Favorable & $7 / 8$ & $88 *$ & - & - \\
Intermediate & $11 / 14$ & 79 & - & $21-75$ \\
Poor & $9 / 16$ & 56 & - & $22-78$ \\
Hematological recovery n= $(33) \S$ & & & & $24-98$ \\
Days to ANC $>500 / \mathrm{mm}^{3}$ & - & - & 30.5 & 35 \\
Days to ANC $>1000 / \mathrm{mm}^{3}$ & - & - & 33 & \\
Hospitalization days & - & - & & \\
\hline
\end{tabular}

ANC: Absolute neutrophil count; CR: Complete response; CRi: incomplete CR; NCCN: National Comprehensive Cancer Network. * One patient lost to follow-up, not considered to be CR. §Of patients who survived to hospital discharge.
Table III. Toxicities and adverse events, $n=38$.

\begin{tabular}{lcccc}
\hline Toxicity/adverse event & $\mathrm{n}$ & $\%$ & Median & Range \\
\hline Mucositis & 12 & 32 & - & - \\
Diarrhea & 29 & 76 & - & - \\
Rash & 23 & 61 & - & - \\
Neutropenic fever & 37 & 97 & - & - \\
ICU admission & 11 & 29 & - & - \\
Documented infection & 22 & 58 & - & - \\
Clostridium difficile infection & 6 & 16 & - & - \\
Bacterial infection & 20 & 53 & - & - \\
Fungal infection & 5 & 13 & - & - \\
Death within 28 days & 4 & 11 & - & - \\
Survival to hospital discharge & 33 & 87 & - & - \\
Patients with diarrhea & & & & \\
$\quad$ Days of diarrhea & - & - & 8 & $1-33$ \\
$\quad$ Days of loperamide & - & - & 2 & $0-15$ \\
Patients with mucositis & & & & $2-48$ \\
$\quad$ Days of mucositis & - & - & 11.5 & $0-21$ \\
$\quad$ Days of mouthwash & - & - & 2 & \\
\hline
\end{tabular}

ICU: Intensive care unit.

pneumonia cases had the following isolates: Candida dubliniensis, C. tropicalis, and Zygomycetes. There was one instance of craniofacial aspergillosis and one of bloodstream infection with $C$. krusei.

Hospitalization, intensive care, and mortality. This study focused on initial hospitalization outcomes only. The median length of hospitalization was 33 days (range $=24-98$ days). Twenty-nine percent of patients required intensive care unit (ICU) admission. Five patients (13.2\%) did not survive to hospital discharge and of these, four died within 28 days, for a treatment-related mortality rate of $11 \%$. Four out of the five deaths during induction therapy were of patients aged 60 years or older $(p=0.34)$. All five patients died with an element of respiratory failure precipitated by cardiac failure at day 15; Stenotrophomonas bacteremia and sepsis at day 20; cirrhosis, encephalopathy and fluid overload at day 21 ; stroke, sepsis and pneumonia day at 21; Candida tropicalis pneumonia and sepsis at day 32 , respectively.

\section{Discussion}

Data from prospective clinical trials show that $60-70 \%$ of patients with AML aged 50 years or less and $50 \%$ of those over 50 years old achieve CR with induction therapy (7). By comparison, IAC at SLU (CR of $71 \%$, median age $=61$ years) was found to be similar in efficacy to historical data, comparing favorably with the PALG DAC studies (median ages 45 and 47 years, respectively) $(5,6)$, and similar to recent reports at other institutions using IAC [median age 58 
years and 43 years] $(10,11)$. However, our study is different from others as our population was older, with 23 patients aged 60 years or more; notably this population was excluded by the PALG and Shen et al. $(5,6,10)$. Consequentially, this retrospective report provides unique information regarding toxicity and induction outcomes in older patients with AML receiving IAC.

Importantly, age stratification did not show differences in efficacy or toxicity in patients 60 years of age or older in this study, despite typically poorer historical CR rates in that group compared to younger patients (7). The CR rate of $65 \%$ among patients 60 years and older in our study is comparable to that reported by Juliusson et al. in which they treated patients over the age of 60 years with intermittent cladribine, intermediatedose cytarabine $\left(1 \mathrm{~g} / \mathrm{m}^{2}\right)$ twice per day for 4 days and 2 days of idarubicin $10 \mathrm{mg} / \mathrm{m}^{2}$ (14). In our hands, IAC did not confer significant $\mathrm{CR}$ advantage for patients with poor cytogenetic classification $(45.5 \%$ vs. $82.6 \%$ with intermediate and $75 \%$ with favorable, $p=0.08$ ) nor when stratified by NCCN leukemia risk $(56 \% v s .79 \%$ with intermediate and $88 \%$ with favorable, $p=0.208$ ). In contrast, the 2012 PALG study reported a trend towards overall survival advantage as well as reduced mortality in patients with unfavorable cytogenetics (6). Shen et al. also showed that IAC was as effective $(\mathrm{CR}=77.8 v \mathrm{~s} .63 \%)$ and tolerable as idarubicin and cytarabine (10). In another retrospective study, 24 patients received IAC (some with lower dose cytarabine) and $79.2 \%$ achieved a CR within 30 days, and 33-month overall survival of $56 \%$ and $36 \%$ disease-free survival was recorded (11).

SLU patient recovery from severe neutropenia $($ ANC $>500$ cells $/ \mathrm{mm}^{3}$ ) was a median of 30.5 days compared to PALG data with DAC, which was a median of 23 (5) and 24 days (6). Shen et al. used lower dose IAC regimen and recorded shorter median time to ANC recovery at 20 days (10).

Skin rash during induction therapy for AML often occurs, but is infrequently described in the literature. We recorded $62 \%$ (23/38) of cases developing skin complaints. Often, the rash was attributed to chemotherapy and was not biopsied. Thirty-five percent $(8 / 23)$ of rashes were biopsied, with one only attributed to chemotherapy. Rash is expected with cytarabine-containing regimens, and in IAC, cladribine may potentiate development of hypersensitivity via a proposed mechanism of lymphopenia and T-cell imbalance (15).

Two studies inform our expectations of gastrointestinal toxicity with $7+3$ induction therapy for AML. Peric et al. found that $41.7-50 \%$ of patients developed generalized oral and gastrointestinal mucositis (16), while Camera et al. revealed that $42 \%$ developed diarrhea during induction (17). We observed oral mucositis in $32 \%(12 / 38)$ and diarrhea in $76 \%$ (29/38) of patients. Our incidence of diarrhea was high, and may have been a result of the additive gastrointestinal toxicity of cladribine and cytarabine, or may represent an overestimate due to our search strategy.
Atallah and colleagues reviewed 1,534 adults with AML to establish expected baseline toxicities with standard induction therapy (18). They showed an overall incidence of documented infection was $64 \%$. The SLU overall documented bacterial infection rate $(53 \%)$ was similar to those seen in PALG studies [37\% (5) and 49\% (6)]. The PALG infection rates did not significantly differ between the $7+3$ and the DAC arms in either study $(5,6)$. However, we recorded $13 \%$ of patients $(5 / 38)$ as having fungal infections compared to $8 \%$ incidence in the PALG study (5).

There are several limitations to this study that should be noted. Because we report a retrospective analysis of the initial induction of 38 patients at a single institution, this study has limited power in ascertaining the performance of the IAC regimen, especially in specific patient subgroups. It is important to note that while not statistically significant, there was a higher incidence of death and $C$. difficile infections noted in the older patient population. Larger trials may have more power to detect differences in outcomes and adverse events. Additionally, our rates of adverse events, such as rash, mucositis and diarrhea, only reflect toxicities that became clinically significant. This may lead to underreporting of toxicities as minor events which may not have been detected or documented in the medical record.

\section{Conclusion}

This report is unique due to the detailed review of toxicities associated with IAC and the older age group of patients. Additionally, SLU uses higher doses of idarubicin and cytarabine in combination with cladribine compared to other published studies $(10,11)$. Several caveats limit the interpretation of this study, namely it is a single-arm, retrospective chart review with a limited number of patients. However, we do show an encouraging $\mathrm{CR}$ rate comparable to prior studies, and no significant differences in remission or incidence of toxicities in patients with AML aged 60 years or more. Cladribine has been implicated in driving increased cytarabine uptake to enhance the potency of this regimen; however, this mechanism may also increase toxicity. Direct comparison of CR, overall survival, and toxicity events after IAC versus standard $7+3$ regimen in older patients would be informative on this issue, especially as part of a prospective randomized trial. For now, however, IAC may be appropriate for AML treatment in selected older patients.

\section{References}

1 Dohner H, Estey EH, Amadori S, Appelbaum FR, Buchner T, Burnett AK, Dombret H, Fenaux P, Grimwade D, Larson RA, Lo-Coco F, Naoe T, Niederwieser D, Ossenkoppele GJ, Sanz MA, Sierra J, Tallman MS, Lowenberg B, Bloomfield CD and European L: Diagnosis and management of acute myeloid leukemia in adults: recommendations from an international 
expert panel, on behalf of the European LeukemiaNet. Blood 115: 453-474, 2010.

2 Wang ES: Treating acute myeloid leukemia in older adults. Hematology Am Soc Hematol Educ Program 2014: 14-20, 2014

3 American Cancer Society: Cancer Facts and Figures 2015. Atlanta, GA, 2015.

4 Yates JW, Wallace HJ Jr., Ellison RR and Holland JF: Cytosine arabinoside (NSC-63878) and daunorubicin (NSC-83142) therapy in acute nonlymphocytic leukemia. Cancer Chemother Rep 57: 485-488, 1973.

5 Holowiecki J, Grosicki S, Robak T, Kyrcz-Krzemien S, Giebel S, Hellmann A, Skotnicki A, Jedrzejczak WW, Konopka L, Kuliczkowski K, Zdziarska B, Dmoszynska A, Marianska B, Pluta A, Zawilska K, Komarnicki M, Kloczko J, Sulek K, Haus O, Stella-Holowiecka B, Baran W, Jakubas B, Paluszewska M, Wierzbowska A, Kielbinski $M$ and Jagoda K: Addition of cladribine to daunorubicin and cytarabine increases complete remission rate after a single course of induction treatment in acute myeloid leukemia. Multicenter, phase III study. Leukemia 18: 989-997, 2004.

6 Holowiecki J, Grosicki S, Giebel S, Robak T, Kyrcz-Krzemien S, Kuliczkowski K, Skotnicki AB, Hellmann A, Sulek K, Dmoszynska A, Kloczko J, Jedrzejczak WW, Zdziarska B, Warzocha K, Zawilska K, Komarnicki M, Kielbinski M, Piatkowska-Jakubas B, Wierzbowska A, Wach M and Haus O: Cladribine, But Not Fludarabine, Added to Daunorubicin and Cytarabine During Induction Prolongs Survival of Patients With Acute Myeloid Leukemia: A Multicenter, Randomized Phase III Study. J Clin Oncol 30: 2441-2448, 2012.

7 NCCN: Acute Myeloid Leukemia. In: NCCN Clinical Practice Guidelines in Oncology. nccn.org: NCCN, 2016.

$8 \mathrm{Li} \mathrm{X}, \mathrm{Xu} \mathrm{S}$, Tan Y and Chen J: The effects of idarubicin versus other anthracyclines for induction therapy of patients with newly diagnosed leukaemia. Cochrane Database Syst Rev 6: CD010432, 2015.

9 Wiernik PH, Banks PL, Case DC, Jr., Arlin ZA, Periman PO, Todd MB, Ritch PS, Enck RE and Weitberg AB: Cytarabine plus idarubicin or daunorubicin as induction and consolidation therapy for previously untreated adult patients with acute myeloid leukemia. Blood 79: 313-319, 1992.

10 Shen Y, Chen J, Liu Y and Wu D: Addition of Cladribine to Idarubicin and Cytarabine during Induction Increases the Overall Efficacy Rate in Adult Patients with Acute Myeloid Leukemia: A Matched-Pair Retrospective Comparison. Chemotherapy 60: 368-374, 2014.
11 Wiedower E, Jamy $\mathrm{O}$ and Martin MG: Induction of Acute Myeloid Leukemia with Idarubicin, Cytarabine and Cladribine. Anticancer Res 35: 6287-6290, 2015.

12 Vardiman JW, Thiele J, Arber DA, Brunning RD, Borowitz MJ, Porwit A, Harris NL, Le Beau MM, Hellstrom-Lindberg E, Tefferi A and Bloomfield CD: The 2008 revision of the World Health Organization (WHO) classification of myeloid neoplasms and acute leukemia: rationale and important changes. Blood 114: 937-951, 2009.

13 Freifeld AG, Bow EJ, Sepkowitz KA, Boeckh MJ, Ito JI, Mullen CA, Raad, II, Rolston KV, Young JA and Wingard JR: Clinical practice guideline for the use of antimicrobial agents in neutropenic patients with cancer: 2010 update by the infectious diseases society of america. Clin Infect Dis 52: e56-93, 2011.

14 Juliusson G, Hoglund M, Karlsson K, Lofgren C, Mollgard L, Paul C, Tidefelt $\mathrm{U}$ and Bjorkholm M: Increased remissions from one course for intermediate-dose cytosine arabinoside and idarubicin in elderly acute myeloid leukaemia when combined with cladribine. A randomized population-based phase II study. Br J Haematol 123: 810-818, 2003.

15 Ganzel C, Gatt ME, Maly A, Ben-Yehuda D and Goldschmidt $\mathrm{N}$ : High incidence of skin rash in patients with hairy cell leukemia treated with cladribine. Leuk Lymphoma 53: 11691173, 2012.

16 Peric KM and Reeves DJ: Tolerability of induction chemotherapy dosing practices in acute myeloid leukemia patients. Leuk Res 39: 173-176, 2015.

17 Camera A, Andretta C, Villa MR, Volpicelli M, Picardi M, Rossi M, Rinaldi CR, Della Cioppa P, Ciancia R, Selleri C and Rotoli $\mathrm{B}$ : Intestinal toxicity during induction chemotherapy with cytarabine-based regimens in adult acute myeloid leukemia. Hematol J 4: 346-350, 2003.

18 Atallah E, Cortes J, O'Brien S, Pierce S, Rios MB, Estey E, Markman M, Keating M, Freireich EJ and Kantarjian H: Establishment of baseline toxicity expectations with standard frontline chemotherapy in acute myelogenous leukemia. Blood 110: 3547-3551, 2007.
Received November 8, 2016

Revised December 15, 2016

Accepted December 20, 2016 